\title{
A New Similarity Measurement Formula between Vague Sets
}

\author{
Jun Jiang \\ Institute of Scientific and Technical Information \\ Chinese Academy of Tropical Agricultural Sciences \\ Danzhou, P. R. of China; \\ College of International Cultural Exchange \\ Qiongzhou University \\ Sanya, P. R. of China \\ daniechyang@126.com \\ Jia Fang \\ Institute of Scientific and Technical Information \\ Chinese Academy of Tropical Agricultural Sciences \\ Danzhou, P. R. of China \\ fang898@126.com
}

\author{
Huijian Zhang* \\ Institute of Scientific and Technical Information \\ Chinese Academy of Tropical Agricultural Sciences \\ Danzhou, P. R. of China \\ sjrdxx@126.com \\ *Corresponding author \\ Hongxu Wang \\ College of Tourism Management \\ Qiongzhou University \\ Sanya, P. R. of China \\ hainanwjs@126.com
}

\author{
Jianchun Guo \\ College of Tourism Management \\ Qiongzhou University \\ Sanya, P. R. of China \\ gjc92@126.com
}

\begin{abstract}
Vague sets can denote and solve more complicated fuzzy and indefinite information. Similarity measure is an important, effective and widely used method on data processing and analysis. Based on the concept of Vague sets and the theory of conversion from single data to Vague data, this research put forward a new similarity measurement formula between Vague sets, to develop the existing Vague sets similarity measurements.
\end{abstract}

Keywords-vague sets; similarity measurement; similarity measurement formula

\section{INTRODUCTION}

The Vague sets theory brought forward by Gau and Buehrer [1] is an extension of Fuzzy sets theory created by Zadeh [2]. Compared with Fuzzy sets theory, Vague sets theory has the ability to denote fuzzy information much more comprehensive.

\section{THE DEFINITION OF VAGUE SETS}

On the assumption that the discourse domain is not empty, to any $u \in U$, define interval $\left[t_{L}(u), 1-f_{L}(u)\right]$ as the Vague membership function or Vague value for Vague set $L$ on point $u \quad$, meantime, $\quad 0 \leq t_{L}(u) \leq 1 \quad, \quad 0 \leq f_{L}(u) \leq 1 \quad$ and $t_{L}(u)+f_{L}(u) \leq 1$.And define $t_{L}(u), \quad f_{L}(u)$ and $\pi_{L}(u)\left(=1-t_{L}(u)-f_{L}(u)\right)$ as real subordinate function, unreal subordinate function and uncertain function of Vague set
$L$ individually. Meanwhile, $t_{L}(u)$ and $f_{L}(u)$ indicate the subordinate extent of supporting and objecting $u \in L$.

When $U=\left\{u_{1}, u_{2}, \cdots, u_{n}\right\}$ is discrete domain, the Vague set $L$ upon that can be expressed as $L=\sum_{i=1}^{n}\left[t_{L}\left(u_{i}\right), 1-f_{L}\left(u_{i}\right)\right] / u_{i}$, or $\quad L=\sum_{i=1}^{n}\left[t_{l_{i}}, 1-f_{l_{i}}\right] / u_{i} \quad$, or $\quad L=\left\{\left[t_{l_{1}}, 1-f_{l_{1}}\right],\left[t_{l_{2}}, 1-f_{l_{2}}\right]\right.$, $\left.\cdots,\left[t_{l_{n}}, 1-f_{l_{n}}\right]\right\}$.

\section{CONVERTING SINGLE VALUE DATA INTO VAGUE DATA}

Building-up a Vague environment is essential to research and solve practical problems through the application of Vague sets theory. And the method is to converting single value data into Vague value data.

Definition 1 on the assumption that $U=\left\{u_{1}, u_{2}, \cdots, u_{n}\right\}$ is the discourse domain, and group $L_{i}(i=1,2, \cdots m)$ is based upon that. Nonnegative single value data $u_{i j}$ are supposed to $u_{j}(j=1,2, \cdots n)$ upon $L_{i}[3]$.

a. Vague axiom, $0 \leq t_{i j} \leq 1-f_{i j} \leq 1$. 
b. Benefit axiom, if $0 \leq u_{k j}<u_{i j}, t_{k j} \leq t_{i j}$ and $1-f_{k j} \leq 1-f_{i j}$, single value data $u_{i j}$ and $u_{k j}$ can be converted into Vague data $L_{i}\left(u_{j}\right)=u_{i j}=\left[t_{i j}, 1-f_{i j}\right]$ and $L_{k}\left(u_{j}\right)=u_{k j}=\left[t_{k j}, 1-f_{k j}\right]$.

If it is satisfied with Vague axiom and Benefit axiom, then the Vague data transformation formula $L_{i}\left(u_{j}\right)=u_{i j}=\left[t_{i j}, 1-f_{i j}\right]$ towards nonnegative single value data $u_{i j}$ is the benefit-type transformation formula from nonnegative single value data $w_{i j}$ to Vague data $V_{i}\left(\mathrm{w}_{j}\right)$.

c. Consumption axiom, if $0 \leq u_{k j}<u_{i j}$, when $t_{k j} \geq t_{i j}, 1-f_{k j} \geq 1-f_{i j}$, single value data $u_{i j}$ and $u_{k j}$ can be converted into Vague data $L_{i}\left(u_{j}\right)=u_{i j}=\left[t_{i j}, 1-f_{i j}\right]$ and $L_{k}\left(u_{j}\right)=u_{k j}=\left[t_{k j}, 1-f_{k j}\right]$.

If it is satisfied with Vague axiom and Benefit axiom, then the Vague data transformation formula $L_{i}\left(u_{j}\right)=u_{i j}=\left[t_{i j}, 1-f_{i j}\right]$ towards nonnegative single value data $u_{i j}$ is the consumption-type transformation formula from non-negative single value data $w_{i j}$ to Vague data $V_{i}\left(w_{j}\right)$.

Above all, the benefit-type transformation formula is fit for project of high extent on both value and preference, while, the consumption transformation formula is fit for project of high extent on value and low extent on preference.

\section{SIMILARITY MEASURE BETWEEN VAGUE SETS}

Definition 2 to any Vague value $l=\left[t_{l}, 1-f_{l}\right]$, define $\quad t_{l}^{(0)}=t_{l} \quad, \quad f_{l}^{(0)}=f_{l} \quad, \quad \pi_{l}^{(0)}=\pi_{l} \quad$; $t_{l}^{(m)}=t_{l} \cdot\left(1+\pi_{l}+\pi_{l}^{2}+\cdots+\pi_{l}^{m}\right)$

$\left.f_{l}^{(m)}=1+\pi_{l}+\pi_{l}^{2}+\cdots+\pi_{l}^{m}\right) \quad, \quad \pi_{l}^{(m)}=\pi_{l}^{m+1} \quad ;$ $\alpha_{l}^{(m)}=t_{l}^{(m)}-f_{l}^{(m)} \quad, \quad \beta_{l}^{(m)}=t_{l}^{(m)}+f_{l}^{(m)}$, $(m=0,1,2, \cdots)[4]$.

Because high differentiate ratio, Vague data mining is widely applied to form similarity measurement formula between Vague sets. The similarity measurement formula between Vague sets below is formed by definition 2 through application of Vague data mining method.

Definition 3 on the assumption that Vague value is $l=\left[t_{l}, 1-f_{l}\right], k=\left[t_{k}, 1-f_{k}\right]$, then the formula $M(l, k)$ is the similarity measure between Vague value $l$ and $k$. Then if $M(l, k)$ is satisfied with the axioms below [5].

a. Commonness axiom, $0 \leq M(l, k) \leq 1$; b. Symmetry axiom, $M(l, k)=M(k, l)$;

c. Reflexivity axiom, $M(l, l)=1$;

d. Minimum axiom, whenever $l=[1,1]$ and $k=[0,0]$, or $l=[0,0]$ and $k=[1,1]$, and $M(l, k)=0$.

$M(l, k)$ means the similarity extent between Vague value $l$ and $k$, the bigger $M(l, k)$ value is, the higher similar level between Vague value $l$ and $k$ is, when $M(l, k)$ value equals to one mean that Vague value $l$ and $k$ are same.

For the other side, the smaller $M(l, k)$ value is, the lower similar level between Vague values $l$ and $k$ is, when $M(l, k)$ value equals to zero mean that Vague value $l$ and $k$ are totally different.

Similar to definition 3, on the basis of similarity measurement $M(L, K)$ between Vague set $L$ and $K$, then the weighted similarity measurement can be defined as $W M(L, K)$.

\section{NEW SIMILARITY MEASUREMENT FORMULA BETWEEN VAGUE SETS}

Theorem 1 If $u_{j \max }=\max \left\{u_{1 j}, u_{2 j}, \cdots, u_{m j}\right\}$, then

$L_{i}\left(u_{j}\right)=u_{i j}=\left[t_{i j}, 1-f_{i j}\right]=\left[\left(\frac{u_{i j}}{u_{j \max }}\right)^{3}, \frac{u_{i j}}{u_{j \max }}\right]$

is the benefit-type transformation formula from nonnegative single value data $u_{i j}$ to Vague data.

While

$L_{i}\left(u_{j}\right)=u_{i j}=\left[t_{i j}, 1-f_{i j}\right]=\left[1-\left(\frac{u_{i j}}{u_{j \max }}\right), 1-\left(\frac{u_{i j}}{u_{j \max }}\right)^{3}\right]$

is the consumption-type transformation formula from nonnegative single value data $u_{i j}$ to Vague data.

Theorem 2 on the assumption that Vague value is $l=\left[t_{l}, 1-f_{l}\right], \quad k=\left[t_{k}, 1-f_{k}\right], \quad m=0,1,2, \cdots$, then

$$
M_{m}(l, k)=\frac{3-\left|f_{l}^{(m)}-f_{k}^{(m)}\right|+\left|\alpha_{l}^{(m)}-\alpha_{k}^{(m)}\right|}{3+\left|f_{l}^{(m)}-f_{k}^{(m)}\right|+\left|\alpha_{l}^{(m)}-\alpha_{k}^{(m)}\right|}
$$


is the similarity measure formula between Vague value $l$ and $k$.

Theorem 3 on the assumption that discourse domain $U=\left\{u_{1}, u_{2}, \cdots, u_{n}\right\}$, upon which there are Vague

sets

$$
L=\sum_{i=1}^{n}\left[t_{L}\left(u_{i}\right), 1-f_{L}\left(u_{i}\right)\right] / u_{i} \quad \text { and }
$$

$K=\sum_{i=1}^{n}\left[t_{K}\left(u_{i}\right), 1-f_{K}\left(u_{i}\right)\right] / u_{i}$, also can be abbreviated as

$L=\sum_{i=1}^{n}\left[t_{u_{i}}, 1-f_{u_{i}}\right] / u_{i} \quad$ and $\quad K=\sum_{i=1}^{n}\left[t_{k_{i}}, 1-f_{k_{i}}\right] / u_{i}$,

meanwhile $m=0,1,2, \cdots$.

Then Formula

$$
T_{m}(L, K)=\frac{1}{n} \sum_{i=1}^{n} \frac{3-\left|f_{l_{i}}^{(m)}-f_{k_{i}}^{(m)}\right|-\left|\alpha_{l_{i}}^{(m)}-\alpha_{k_{i}}^{(m)}\right|}{3+\left|f_{l_{i}}^{(m)}-f_{k_{i}}^{(m)}\right|-\left|\alpha_{l_{i}}^{(m)}-\alpha_{k_{i}}^{(m)}\right|}
$$

is the similarity measure formula between Vague sets $L$ and $K$.

Theorem 4 on the assumption that $w_{i} \in[0,1]$ is the weight of element $u_{i}(i=1,2, \cdots, n)$, and $\sum_{i=1}^{n} w_{i}=1$, when $m=0,1,2, \cdots$.

Then upon the assumption of theorem 3, formula

$$
W T_{m}(L, K)=\sum_{i=1}^{n} w_{i} \cdot \frac{3-\left|f_{l_{i}}^{(m)}-f_{k_{i}}^{(m)}\right|-\left|\alpha_{l_{i}}^{(m)}-\alpha_{k_{i}}^{(m)}\right|}{3+\left|f_{l_{i}}^{(m)}-f_{k_{i}}^{(m)}\right|-\left|\alpha_{l_{i}}^{(m)}-\alpha_{k_{i}}^{(m)}\right|}
$$

is the weighted similarity measure formula between Vague sets $L$ and $K$.

\section{CONCLUSION}

The above-mentioned similarity measurement formula between Vague sets can be applied to select product series as per the selector's desire.

The applicable steps are as follows. First of all, the products to be selected and the indexes upon which should be found. Secondly, the candidates to be selected need to be decided. Thirdly, the ideal product should be decided. Then raw data needed to be converted into Vague data, meanwhile, the Vague sets between the ideal product and candidates are established. Soon after, the weight of each index should be decided as per the selector's desire. After this, the weighted Vague sets similarity measure between the ideal product and candidates need to be calculated. Finally, the most desirable product is found.
This similarity measurement formula between Vague sets is a special example of Vague pattern recognition, can be used to make decisions between multiple targets.

\section{ACKNOWLEDGMENT}

This research is sponsored by Chinese Agriculture Ministry fund bearing No. 201403075.

\section{REFERENCES}

[1] Gau W L, Buehrer D J. "Vague sets", IEEE Transaction on Systems[J].Man and Cybernetics,1993:23(2): 610-614.

[2] Zadeh L A, "Fuzzy sets", Information and Control, Shenyang, China, 1965: (8):pp338-356.

[3] Wang Hong-xu. Definition and transforming formulas from the single valued data to the vague valued data[J].Computer Engineering and Applications, 2010,46(24):42-44.

[4] Liu Hua-wen, Wang Feng-ying. Transformations and Similarity Measures of Vague Sets [J].Computer Engineering and Applications, 2004, 40(32):79-81, 84.

[5] Wang Hong-xu. Apply Vague Optimized Decision-Making Method for Comprehensive Evaluation of New Wheat Varieties [J].Computer Engineering and Applications, 2011, 47(12):210-212. 